\title{
Methodological approach for assessing the cost-effectiveness of treatments using longitudinal observational data: The SOHO study
}

\author{
Frank Windmeijer \\ University of Bristol
}

\section{Stathis Kontodimas}

Eli Lilly and Company

\author{
Martin Knapp \\ London School of Economics and Political Science
}

\section{Jacqueline Brown}

Eli Lilly and Company

\section{Josep Maria Haro \\ Fundació Sant Joan de Déu}

Objectives: The objective of this study was to develop a method to allocate treatment effects when patients switch medication frequently in longitudinal observational studies and apply the approach to assess the cost-effectiveness of treatments in the Schizophrenia Outpatient Health Outcomes (SOHO) study.

Methods: Data were collected on patients at entry to the SOHO study at 3, 6, and 12 months. The 12-month follow-up period was considered as three epochs: 0-3 months, 3-6 months, and 6-12 months. Patients who switched treatment at 3 months had their new treatment considered as a new baseline observation, as these two 3-month observations provide two sets of information on the cost-effectiveness of a drug in the first 3 months after initiation. Multivariate regression analysis was used to adjust for baseline covariates. The model allowed for flexible functional forms, and the cost data were

\footnotetext{
The Schizophrenia Outpatient Health Outcomes (SOHO) study is funded by Eli Lilly and Company, Windlesham, Surrey, UK. The SOHO study group: (France) J.-P. Lepine, Hôspital Fernand Widal, Paris; I. Gasquet, Hôpital Cochin, Paris; (Germany) D. Naber, Universitatskrankenhaus-Eppendorf, Hamburg; (the Netherlands) C.J. Slooff, Kenniscentrum Schizofrenie, Assen; (Spain) J. Alonso, University of Barcelona, Barcelona; J.M. Haro, Sant Joan de Deu-SMM, St. Boi, Barcelona; (UK) T. Croudace and P.B. Jones, Addenbrooke's Hospital, Cambridge; M. Knapp, PSSRU, London School of Economics, London. We thank at Eli Lilly and Company, Mark Belger for his statistical advice, Jo Cooper for her administrative support, and Diego Novick, Spyridon Tzivelekis, and Mark Ratcliffe for their comments.

Declaration of interest

Frank Windmeijer received monetary compensation from Eli Lily and Company for econometric advice. Martin Knapp and Josep Maria Haro were paid by Eli Lily and Company for their participation in the SOHO Advisory Board. Stathis Kontodimas and Jackie Brown are Eli Lilly and Company employees.
} 
modeled using an exponential mean function. Bootstrapping assessed the uncertainty of the estimated parameters and incremental cost-effectiveness analysis decision rule.

Results and Conclusions: We show the feasibility of the epoch analysis approach using data from the SOHO study comparing two antipsychotics. Estimates for the incremental cost and effectiveness per epoch over the full 12-month period are presented. Using the estimates of 200 bootstrap samples, we demonstrate how one drug is cost-effective compared with another.

\section{Keywords: Observational studies, Cost-effectiveness, Longitudinal data, Epoch analysis}

Randomized controlled trials (RCTs) are the gold standard for assessing efficacy and safety of healthcare technologies, including pharmaceutical products. They can demonstrate whether a healthcare intervention works in a defined population and have a high internal validity, as patients are randomly allocated to protocol-driven treatments with strict study entry criteria. However, these same features may restrict the generalizability of the findings to the everyday clinical setting. Government agencies, such as the French Transparency Committee, therefore, request that pharmaceutical companies demonstrate the effectiveness of their products in an everyday clinical setting. Observational studies are, thus, a useful nonexperimental complement to RCTs (9).

Epidemiological observational studies that longitudinally follow-up cohorts of individuals in a naturalistic setting without randomization to treatment, have traditionally been performed to determine whether there is an association between disease status (e.g., heart disease) and exposure to certain factors (e.g., cholesterol level or smoking) (17). This type of study design, however, can also be used to determine whether there is an association between the drugs administered and their effectiveness.

Data showing the cost-effectiveness of pharmaceutical products are also increasingly required for access and reimbursement, particularly in the United Kingdom, Canada, Australia, Sweden, and the Netherlands. Observational studies are a useful vehicle for determining whether there is a difference in the costs (and hence cost-effectiveness) associated with the drugs administered outside of the experimental setting.

Nonrandomized studies are more prone to bias, however. They are at particular risk of selection bias if investigators select patients for treatments on the basis of the characteristics of the patient or their disease, thus leading to systematic differences between comparison groups (4).

The convention is to adjust for differences in risk or prognostic factors to avoid confounding. This strategy assumes that the researchers know the most important prognostic factors and that these factors have been measured appropriately in the study (3). Even if appropriate adjustments can be made, observational studies still pose methodological problems, as in everyday clinical practice patients may be prescribed more than one medication and frequently switch medications. This finding raises the issue of how to attribute the medication effects to the outcomes of interest. This study describes a novel method of analysis, the so-called epoch analysis, to address this issue. It uses the economic analysis conducted as part of the Schizophrenia Outpatient Health Outcomes (SOHO) study as an illustrative case $(6 ; 7)$, where the specific outcomes of interest are the incremental cost and quality-adjusted life-years (QALYs) gained as derived from the health-related quality of life instrument EQ-5D (EuroQol) (2).

The proposed method of analysis allows alternative estimation approaches to be used to take into account the skewed nature of the cost data (10). It uses an adaptation of the Manning and Mullahy (10) method for choosing among the alternative estimators and uses the Poisson estimator to estimate the cost models.

\section{SOHO and Schizophrenia}

The European SOHO study is the largest naturalistic study of antipsychotic drugs conducted to date (6;7). It is a 3-year, prospective, observational study of the outcomes of antipsychotic treatment for schizophrenia in the outpatient setting. One of the main objectives of SOHO is to understand the comparative costs and outcomes of therapy for schizophrenia with olanzapine versus other antipsychotic medications in a clinical setting.

The SOHO study is being conducted in ten European countries with 1,096 investigators participating. A total of 10,972 patients with schizophrenia starting or changing medication in the outpatient setting have been enrolled in SOHO, undergoing evaluation at regular time intervals (at enrollment and at 3, 6, 12,18, 24, 30, and 36 months). Data collected include patients' prognostic characteristics and other potential confounding characteristics; health service resource use such as medication use, the number of hospital inpatient and outpatient visits; and patient self-reported EQ-5D health state classifications (2). Details of the study methods have been published elsewhere $(6 ; 7)$.

All patient care was at the discretion of the participating psychiatrist; no specific instructions were included in the study description. SOHO is essentially observing a window of 3 years of care in the patient's schizophrenia treatment path. 
This study focuses on the economic analysis for the first 12 months. As expected (3), outside of the experimental setting of an RCT, a sizeable proportion of patients (31 percent) switched their antipsychotic medication during follow-up or added another antipsychotic medication to their baseline treatment.

\section{METHODS}

\section{Allocation of Treatment Effects}

The objective of the analysis is to estimate the treatment effects on costs and change in EQ-5D tariff or utility score (2) of the drugs taken by the patients. Difficulties arise with conventional methods of allocating and interpreting such effects when patients frequently switch medication. With intentionto-treat analysis, the recommended method for analyzing RCTs, the treatment effects are attributed to the medication given at study entry. When patients frequently change medication, this type of analysis could favor less effective medications at study entry if patients are subsequently switched to more effective medications.

The treatment effects of each drug could be allocated in proportion to the duration of treatment on the drug, as in an exposure approach. For example, if drug A was taken for 3 months and drug B subsequently for 9 months, then over the 12-month period, 25 percent of the 12-month effectiveness would be attributed to drug $\mathrm{A}$ and 75 percent to drug $\mathrm{B}$. This strategy assumes effectiveness is additive and linear with duration, which may not be realistic. The linearity assumption of course can easily be relaxed, but it is not clear what the appropriated functional form would be.

A new method of analysis, thus, was needed to attribute treatment effects directly to the antipsychotic medication taken by the patients. For the purposes of this data analysis, we divided the data into three epochs: 0-3 months, 3-6 months, and 6-12 months (hence the name epoch analysis). To maximize the data used, a patient may contribute more than one observation per epoch. Each epoch provides information on the treatment effects during the respective time period.

Using a hypothetical example, we describe here how the treatment effects are assigned using the epoch analysis. During the 12 months of data collection, we have four observations per patient (enrollment, 3, 6, and 12 months after enrollment, referred to below as $t=1,2,3$, and 4).

For simplicity, assume that a patient can receive one of two drugs during the observation period: drug A or drug B. Imagine that a patient receives drug A for the whole 12-month observation period (see Figure 1a). The first epoch provides information on the treatment effect of drug A over the first 3 months of treatment. The second epoch provides information on the treatment effect of drug A over the subsequent 3-month period of treatment conditional on being treated with drug A during the first 3 months of treatment. The third epoch provides information on the treatment effect of drug A over the last six months conditional on having been treated with drug A during the previous 6 months. Being on the same treatment in the prior period is instrumental as it provides the necessary conditions for combining the three treatment effects to provide a cumulative treatment effect over the 12-month period.

Now imagine that a second patient switches to drug $\mathrm{B}$ after 3 months of treatment with drug A (see Figure 1b). The first 3 months of treatment with drug A contributes to the first epoch, depicted as Epoch 1A. The patient, however, initiates a new medication at time $t=2$, which is effectively a new baseline observation; hence, the first 3 months of drug B contribute to the first epoch, depicted as Epoch 1B. For this patient, two observations contribute to the first epoch: one for drug A and one for drug B. In this case, data from the second 6-month observation period cannot be analyzed as Epoch 2 for drug B as the time period is not consistent with the analysis ( 3 months).

A third patient switches medication from drug A to drug $\mathrm{B}$ at observation $t=3$ (see Figure 1c). For this patient, Epoch 1 and Epoch 2 are used as for patient one. Data from the second 6-month observation period cannot be analyzed as Epoch 1B, however, as the time period is not consistent with the analysis (3 months).

As pointed out above, some observation periods for various patient types are not being used for the estimation of the treatment effects due to the unequal time periods. As further observation periods become available with the SOHO data, a more efficient use of the patient observations may be to analyze the data in 6-month epochs. However, as most of the benefits of antipsychotic medications tend to occur during the first 4 to 6 weeks of treatment, we decided to keep the duration of the first two epochs as 3 months for the 12-month data analysis.

\section{Estimation Issues}

Multivariate regression analysis was used to examine the impact of treatment on costs and EQ-5D tariff, adjusting for confounding effects to take account of selection bias (4). To allow for flexible treatment effects over time, we estimate separate coefficients for the different epochs. The three regression models used to model costs and outcomes are:

First Epoch. Denote $Y_{i t, 3}$ as the dependent variable, cost or change in EQ-5D tariff, during the first 3-month epoch for patient $i$ at time $t, t \in\{2,3\}$. The multivariate model is specified as:

$$
\begin{aligned}
Y_{i t, 3}= & f\left(x_{i, t-1}^{\prime} \beta_{3}+\sum_{j=1}^{J} \delta_{j 3} D_{i j t}\right. \\
& \left.+\sum_{k=2}^{3} \alpha_{k 3} M_{i k t}+\gamma S_{i t}+u_{i t, 3}\right), \quad t \in\{2,3\}
\end{aligned}
$$


(a)

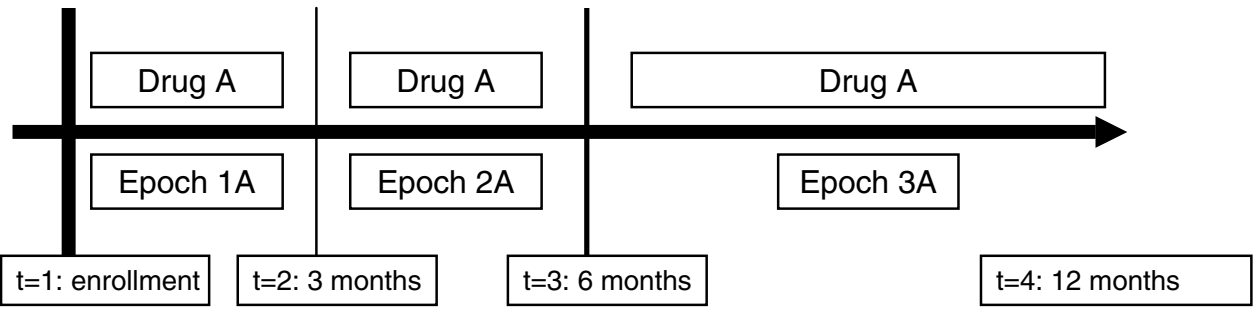

Epoch 1A: Epoch 1 for drug A Epoch 2A: Epoch 2 for drug $A$ Epoch 3A: Epoch 3 for drug A

(b)

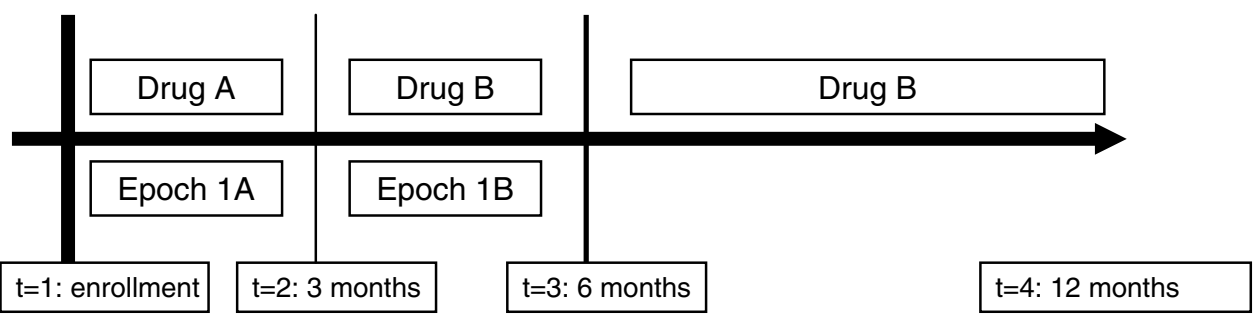

Epoch 1A: Epoch 1 for drug A Epoch 1B: Epoch 1 for drug $B$

(c)

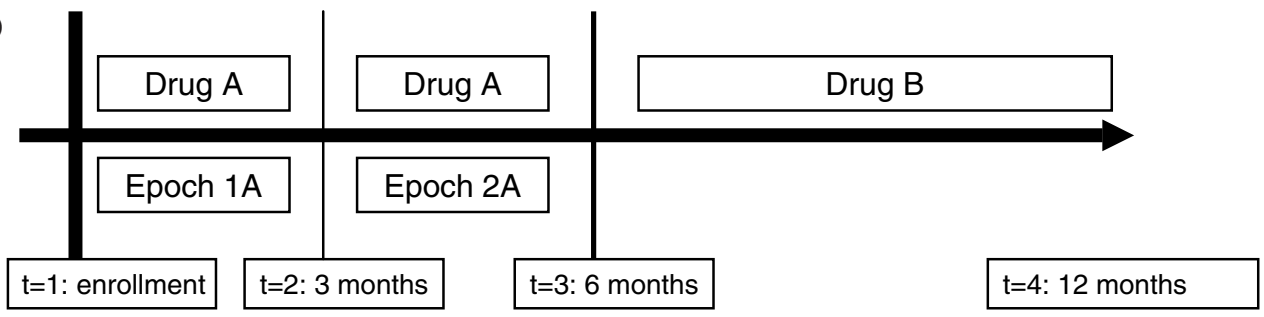

Epoch 1A: Epoch 1 for drug A

Epoch 2A: Epoch 2 for drug $A$

Figure 1. Epoch analysis. (a) Patient who stays on the same medication over 12 months. (b) Patient who switches medication after the first 3 months. (c) Patient who switches medication after 6 months.

where $D_{i j t}$ is the treatment indicator, $D_{i j t}=1$ when a patient is prescribed drug $j$ and 0 otherwise, $\beta_{3}$ measures the effects of the baseline covariates, and $\delta_{j 3}$ are the incremental treatment effects on cost or change in EQ-5D tariff relative to the excluded treatment, which we will refer to as drug B. The variable $M_{i k t}$ is an indicator variable for multiple drug use. $M_{i k t}=1$ when a patient takes $k$ drugs simultaneously, with $k$ equal to 2 or 3 . The indicator variable $S_{i t}=1$ when a patient has switched medication after 3 months, and for those patients we include two observations. The vector of confounders $x$ contains information on variables, including age, gender, and country of residence of the patients, and various comorbidity measures. It further includes a full set of investigator indicators to allow for possible differential investigator effects that may be correlated with prescription choices. The full list of baseline covariates is given in Table 1 . For those patients that do not switch medication at $t=2$ and, therefore, have one observation in the first epoch, $x_{i, t-1}$ are the confounders measured at the first visit. For those who switch medication at $t=2, x_{i, t-1}$ measures the confounders at baseline for the first observation period, but for the second observation, it measures the confounders observed at the second visit $t=2$.

Second Epoch. Denote $Y_{i 3,3 \mid 3}$ as the dependent variable for the second 3-month epoch, given that the patient already had 3 months of continuous treatment on the same medication as that in the second epoch. The model is then specified as: 
Table 1. List of Baseline Covariates Used for Adjustment in the Models

- Age upon presentation

- Age squared

- Age at first service contact for schizophrenia

- Suicide attempts 6 months before enrollment

- Body mass index at previous visit/baseline

- Weight in kilograms at baseline

- Variable indicating whether the patient is using antipsychotics for the first time

- Treatment received 6 months before baseline

- Receiving an antipsychotic upon presentation to the baseline visit

- Receiving mood stabilisers upon presentation

- Positive symptoms at baseline (CGI)

- Negative symptoms at baseline (CGI)

- Cognitive symptoms at baseline (CGI)

- Depressive symptoms at baseline (CGI)

- Overall symptom severity at baseline (CGI)

- Presence of extrapyramidal symptoms at baseline

- Tardive dyskinesia at baseline

- Loss of libido at baseline

- Presence of amenorrhea/other menstrual disturbance at baseline

- Presence of impotence/sexual dysfunction at baseline

- Patient's compliance/adherence to prescribed antipsychotic therapy during the past 4 weeks before baseline

- Substance dependency and/or abuse 4 weeks before baseline

- Alcohol abuse/dependency 4 weeks before baseline

- Receiving monotherapy or combination treatment at baseline

- Psychiatrist indicators

- Employment status in the 4 weeks before recruitment

- Gender

- Housing status in the 4 weeks before recruitment

- Resource use 6 months before enrollment (only for cost models)

- Score of dependent variable at baseline

CGI, Clinical Global Impression scale.

$$
Y_{i 3,3 \mid 3}=f\left(x_{i 1}^{\prime} \beta_{3 \mid 3}+\sum_{j=1}^{J} \delta_{j 3 \mid 3} D_{i j 3}+\sum_{k=2}^{3} \alpha_{k 3 \mid 3} M_{i k t}+u_{i 3,3 \mid 3}\right)
$$

where $\beta_{3 \mid 3}$ measures the effects of the baseline covariates, and $\delta_{j 3 \mid 3}$ are the incremental treatment effects on costs or changes in EQ-5D tariff.

Third Epoch. Denote $Y_{i 4,6 \mid 6}$ as the dependent variable for the third epoch, a 6-month period, given that the patient already had 6 months of continuous treatment on the same medication. The model is then specified as:

$$
Y_{i 4,6 \mid 6}=f\left(x_{i 1}^{\prime} \beta_{6 \mid 6}+\sum_{j=1}^{J} \delta_{j 6 \mid 6} D_{i j 4}+\sum_{k=2}^{3} \alpha_{k 6 \mid 6} M_{i k t}+u_{i 4,6 \mid 6}\right)
$$

where $\beta_{6 \mid 6}$ measures the effects of the baseline covariates, and $\delta_{j 6 \mid 6}$ are the incremental treatment effects.

When the dependent variable is the change in EQ-5D tariff data, the models are estimated using ordinary least squares (OLS), that is, a linear $f$ for each of the three epochs. When the dependent variable is cost, $f$ is specified as an exponential mean function to take account of the skewed distribution of the cost data. Although the cost data are clearly nondiscrete, the parameters can be consistently estimated using standard Poisson regression models. A modified Park (15) test developed by Santos Silva and Tenreyro (18) indicated that this specification is adequate for these data relative to other types of distributions, such as the Gamma or inverse-Gaussian. Also, unlike the latter, the Poisson specification gives equal weight to extreme values (18), which is advantageous as data from patients that are high resource users should be given equal weight to those from low resource users.

\section{Incremental Cost and QALYs Gained over 12 Months}

When an exponential function is used, the estimated treatment effects are multiplicative such that $\left(\exp \left(\hat{\delta}_{j}\right)-1\right)$ gives the estimated percentage change in treatment cost. To calculate the incremental cost, we took as a reference cost the average predicted cost for the sample as a whole, assuming that all patients were drug B users. For example, for the first epoch:

$$
\hat{C}_{3}^{B}=\frac{1}{N} \sum_{i=1}^{N} \exp \left(x_{i, t-1}^{\prime} \hat{\beta}_{3}+\hat{\gamma} S_{i t}\right)
$$

where $\hat{\beta}_{3}$ and $\hat{\gamma}$ are the estimated coefficients. The incremental costs for drug $j$ are then calculated as $\left(\exp \left(\hat{\delta}_{j 3}\right)-1\right) \hat{C}_{3}^{B}$. The total cost differentials over the 12-month period can be obtained by adding up the incremental costs figures over the three epochs.

As discussed above, when the dependent variable is the change in EQ-5D tariff data, the models are linear and estimated by OLS. To estimate the QALYs gained using the coefficients from the models for change in EQ-5D, the additional QALYs gained over the 12-month period for drug $j$ relative to drug $\mathrm{B}$ were approximated by:

$$
\begin{aligned}
& \text { QALYgained over } 12 \text { months } \\
& =\left((3 / 12) \times \hat{\delta}_{j 3}\right) \times 0.5 \text { for the first epoch } \\
& \left.+(3 / 12) \times \hat{\delta}_{j 3}\right)+\left((3 / 12) \times \hat{\delta}_{j 3 \mid 3}\right) \\
& \times 0.5 \text { for the second epoch } \\
& +(6 / 12) \times\left(\hat{\delta}_{j 3}+\hat{\delta}_{j|3| 3}\right)+\left((6 / 12) \times \hat{\delta}_{j 6 \mid 6}\right) \\
& \times 0.5 \text { for the third epoch }
\end{aligned}
$$

The above numerical presentation is equivalent to measuring the area under the curve of the difference in treatment effects (see Figure 2).

For the cost and change in EQ-5D models, adding up the effects was only possible as the models for the later periods were conditional on the patient having continuous treatment up to the point of analysis. The incremental cost-effectiveness ratio (ICER) was finally estimated as the incremental costs, as estimated above, divided by the QALYs gained over the 12-month period. 
Key: $\quad \hat{\delta}_{j 3}=$ incremental change in $\mathrm{EQ}-5 \mathrm{D}$ tariff between drug $\mathrm{j}$ and drug $\mathrm{B}$ during first epoch

$\hat{\delta}_{j 3 \mid 3}=$ incremental change in EQ-5D tariff between drug $j$ and drug $B$ during second epoch

$\hat{\delta}_{j 66}=$ incremental change in EQ-5D tariff between drug j and drug B during third epoch

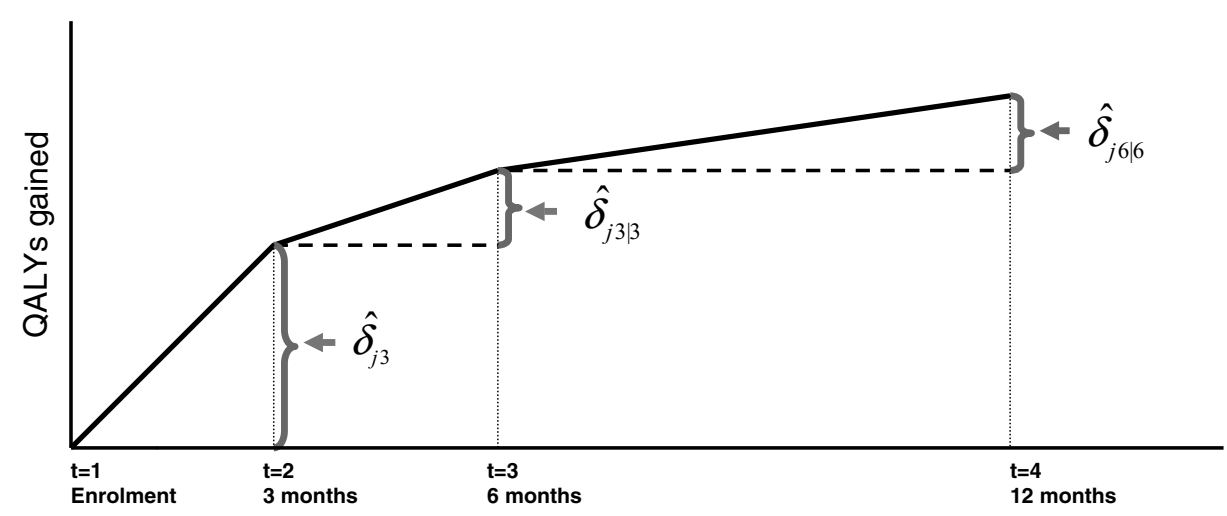

Figure 2. Estimating quality-adjusted life-years (QALYs) gained from model coefficients.

\section{Dealing with Uncertainty}

Uncertainty in the analysis can be addressed using conventional statistical techniques such that standard errors of the $\hat{\delta}$ coefficients and the associated confidence intervals can be estimated for each of the regression models to show parameter uncertainty within each epoch.

Uncertainty in the sampling was addressed by using nonparametric bootstrapping to replicate the incremental treatment effects. Two hundred samples were sampled randomly, with replacement from the data of each patient, each sample providing data to estimate the corresponding incremental cost and QALYs gained (1). Uncertainty in the ICER decision rule was addressed using the bootstrap replications of incremental costs and utilities to calculate the cost-effectiveness acceptability curve of drug A compared with drug B. Each replication of the incremental costs and utilities was used to re-estimate the ICER. The probability that drug A is costeffective compared with drug B was estimated as the proportion of replicated ICER falling below a specified value assigned to the willingness to pay for a QALY gained. For example, the National Institute of Clinical Excellence threshold for the willingness to pay for QALY gained is around $£ 30,000$ (5).

\section{RESULTS}

We present an example of the above methodological framework, whereby the incremental cost and additional QALYs gained are estimated for one antipsychotic, drug A, relative to drug B, using the first 12 months of data on resource use and EQ-5D tariffs collected within the SOHO study.
For purposes of this study, we have used UK unit costs for medication use, hospital inpatient days, psychiatric sessions and day care. These unit costs were taken from published sources (11-14) and applied to volume of resource use taken from the pan-European SOHO data. The treatment assigned to the epoch was assumed to be the medication received immediately before the assessment visit. Table 2 gives the incremental treatment effects on costs and changes in EQ-5D tariff estimated for drug A compared with drug $\mathrm{B}$ for each of the epochs. The results show that the total treatment costs for patients using drug A are on average $£ 81$ higher than those for patients treated with drug $B$ in the first epoch, although there is quite a large uncertainty attached to this finding, as indicated by the large bootstrap standard error of 58. In contrast, for the second and third epochs, the total treatment costs for patients using drug A are on average $£ 114$ and $£ 157$ lower, respectively, than for patients using drug B. Over the 12-month period, the total treatment costs are, therefore, on average $£ 190$ lower for patients using drug $\mathrm{A}$, with a bootstrap standard error of 130 .

The estimation results of the model for changes in EQ$5 \mathrm{D}$ show that drug $\mathrm{A}$ is less effective than drug $\mathrm{B}$ for the first 3-month epoch. The estimated coefficient is -0.0443 with a very small bootstrap standard error of 0.0066 , indicating that this finding is a statistically significant incremental difference. For the second epoch, the estimated $\hat{\delta}_{A 3 \mid 3}$ is very close to zero, -0.0034 , and not significant. In the third epoch, drug $\mathrm{A}$ is again marginally less effective, with an estimated incremental difference of $\hat{\delta}_{A 6 \mid 6}=-0.0117$, with a bootstrap standard error of 0.0077 . The resulting incremental QALYs lost for drug A users over the 12-month period is 
Windmeijer et al.

Table 2. Estimation Results

\begin{tabular}{|c|c|c|c|c|}
\hline $\begin{array}{l}\text { Cost model (dependent } \\
\text { Poisson regression }\end{array}$ & $\begin{array}{l}\text { riable: total treatment costs) } \\
\text { First epoch }(0-3 \text { months }) \\
\quad \exp \left(\hat{\delta}_{A 3}-1\right) \hat{C}_{3}^{B}\end{array}$ & $\begin{array}{l}\text { drug A versus drug B } \\
\text { Second epoch }(3-6 \\
\text { months }) \exp \left(\hat{\delta}_{A 3 \mid 3}-1\right) \hat{C}_{3 \mid 3}^{B}\end{array}$ & $\begin{array}{l}\text { Third epoch (6-12 months) } \\
\quad \exp \left(\hat{\delta}_{A 6 \mid 6}-1\right) \hat{C}_{6 \mid 6}^{B}\end{array}$ & Sum \\
\hline No. of observations ${ }^{\mathrm{a}}$ & 6152 & 4356 & 3496 & \\
\hline Coefficient & 81.44 & -114.13 & -157.46 & -190.15 \\
\hline Bootstrap standard error & 57.61 & 47.54 & 81.71 & 130.04 \\
\hline $95 \% \mathrm{CI}$ & $-31.48,194.36$ & $-207.32,-20.95$ & $-317.62,2.71$ & $-445.03,64.73$ \\
\hline \multicolumn{5}{|c|}{ Change in EQ-5D model (dependent variable: $\triangle \mathrm{EQ}-5 \mathrm{D}$ ), drug A versus drug $\mathrm{B}$} \\
\hline OLS & $\begin{array}{c}\text { First epoch (0-3 months) } \\
\hat{\delta}_{A 3}\end{array}$ & $\begin{array}{l}\text { Second epoch (3-6 } \\
\text { months) } \hat{\delta}_{A 3 \mid 3}\end{array}$ & $\begin{array}{c}\text { Third epoch (6-12 months) } \\
\hat{\delta}_{A 6 \mid 6}\end{array}$ & QALY \\
\hline No. of observations ${ }^{a}$ & 8303 & 5881 & 4730 & \\
\hline Coefficient & -0.0443 & -0.0034 & -0.0117 & -0.0438 \\
\hline Bootstrap standard error & 0.0066 & 0.0070 & 0.0077 & 0.0057 \\
\hline $95 \% \mathrm{CI}$ & $-0.0573,-0.0313$ & $-0.0171,0.0103$ & $-0.0267,0.0033$ & $-0.0551,-0.0326$ \\
\hline
\end{tabular}

${ }^{a}$ For full sample, including patients using other drugs.

CI, confidence interval; OLS, ordinary least squares; QALY, quality-adjusted life-years.

estimated to be -0.0438 , with a small bootstrap standard error of 0.0057 .

Combining the results of the cost and effectiveness models, we find that the incremental cost of drug B over the 12 months is $£ 190$ and the QALYs gained are 0.0438 , giving an incremental cost per QALY gained of $£ 4,341$. Figure 3 presents the incremental cost-effectiveness plane using the incremental cost and effectiveness estimates from the 200 bootstrap samples. These give a good indication of the uncertainty associated with the incremental cost per QALYs gained. Also shown for the purpose of illustration is the $£ 30,000$ per QALY gained threshold. If this threshold is taken as society's willingness to pay for a QALY gained, then the probability that drug B is cost-effective relative to drug $\mathrm{A}$ is 1 , as all the bootstrap ICER estimates show the incremental cost per QALY gained to be below the $£ 30,000$ threshold.

The cost-effectiveness acceptability curve depicted in Figure 4 shows the probability of drug B being cost-effective compared with drug A. The probability of being costeffective increases to 1 for values of around $£ 16,000$ per QALY gained or more.

\section{DISCUSSION}

Observational studies offer the opportunity to study patients in the everyday clinical setting. Unlike RCTs, where the treatments are protocol driven and there are strict entry criteria, observational studies can reflect the complexity of everyday clinical practice. The course of treatments within the observation period, thus, can vary significantly between different patients or practices. This finding is important in schizophrenia where it is well known that patients change medication frequently (3).
In economic evaluation, the effects of each drug or treatment on costs and effectiveness have to be estimated. This study presents a methodological framework that allows the treatment effects to be estimated in a longitudinal observational study where some patients have switched their medication or treatment.

The proposed epoch analysis is possible as the observational study is a "window" in the patients' treatment pathway. In the SOHO study, switching medication within the study is equivalent to the entry criteria into the study. Switching medication, thus, is essentially equivalent to a new baseline measurement. However, it assumes that there is no carry over of effects into the following epoch, which effectively means that the effects of the medication wear off as soon as the medication is discontinued.

One of the main advantages of the epoch analysis approach is that it is able to use data from all types of patients; from those who remain on the same therapy throughout follow-up to those patients who took a combination of medications and/or change medication.

Combination treatments are not modeled as separate treatments. Instead, if a patient is taking more than one antipsychotic treatment, then the effects are attributed to each of the treatments received, and a "correction" combination coefficient is fitted in the model by incorporating binary indicator variables for combinations of two or three antipsychotics.

To control for the fact that the patients with repeated observations for the first epoch may be inherently different from those patients who do not switch treatment, a switching/repeated observation binary indicator is fitted in the models. The proposed approach allows for an accurate measurement of the short-term effects of a medication and provides the opportunity to use this information when estimating the cumulative measure of effectiveness of treatment. This feature is important in schizophrenia as it is expected that most of 


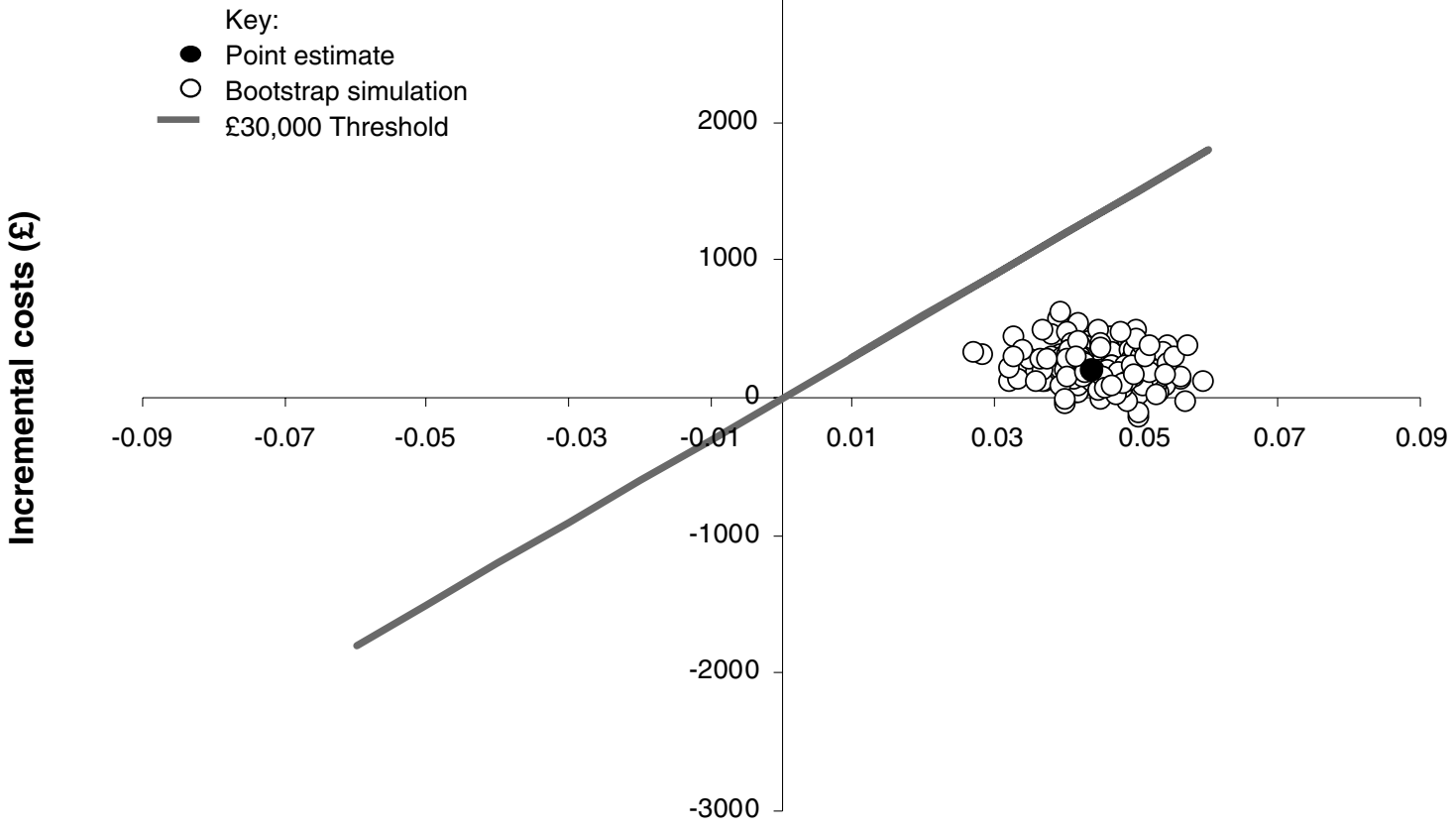

Incremental Quality Adjusted Life Years (QALYs)

Figure 3. Incremental cost effectiveness plane showing drug B versus drug A. QALYs, quality-adjusted life-years.

the improvements in clinical status will occur during the first months after initiating or switching treatment. Thereafter, the patients will most likely enter a maintenance treatment phase (8).

The study also shows that the proposed framework allows the application of accepted methods in the econometrics literature to take into account the skewed nature of cost data
(10). The epoch analysis is also flexible enough to allow for a reliable representation of uncertainty in sampling using nonparametric bootstrap resampling and uncertainty in the decision rule by means of the cost-effectiveness acceptability curve (1).

In this study, we use UK unit costs and apply these costs to the pan-European resource use data. It is recognized,

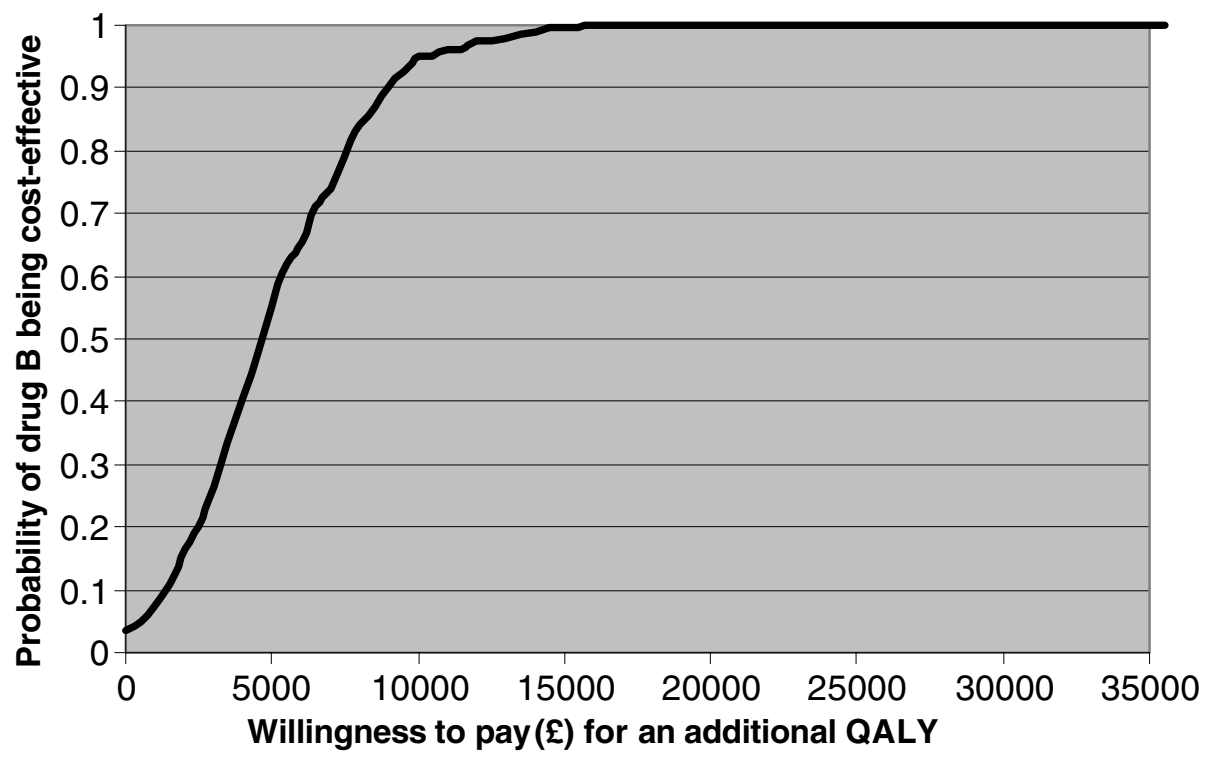

Figure 4. Cost-effectiveness acceptability curve for drug B versus drug A. QALY, quality-adjusted life-year. 
however, that there is ongoing debate in the literature regarding the appropriate method of aggregating unit costs in multinational studies $(16 ; 19)$.

Effectiveness and cost-effectiveness data from patients in everyday clinical settings is becoming increasingly important for reimbursement and access decisions. Longitudinal observational studies provide a vehicle to address these data needs. However, traditional methods of analysis are not adequate when it comes to assigning treatment effects to the drugs taken by patients when there is a tendency for them to switch their medication frequently. We have proposed the epoch analysis to address this issue. The approach is flexible enough to incorporate current methods to address the modeling of skewed cost data, sampling, and decision-making uncertainty.

\section{CONTACT INFORMATION}

Frank Windmeijer, PhD (f.windmeijer@bristol.ac.uk), Department of Economics, University of Bristol, 8 Woodland Road, Bristol BS8 1TN , UK

Stathis Kontodimas, MSc (kontodimas_stathis@lilly.com), Senior Health Economist, European Health Outcomes Research, Eli Lilly and Company, Erl Wood Manor, Sunninghill Road, Windlesham, Surrey GU20 6PH, UK

Martin Knapp, PhD (m.knapp@LSE.ac.uk), Professor of Social Policy, Personal Social Services Research Unit, London School of Economics \& Political Science, Houghton Street, London WC2A 2AE, UK

Jacqueline Brown, PhD (jackie.brown@lilly.com), CNS Team Leader, European Health Outcomes Research, Eli Lilly and Company, Erl Wood Manor, Sunninghill Road, Windlesham, Surrey GU20 6PH, UK

Josep Maria Haro, PhD (jmharo@comb.es) Director, Sant Joan de Déu - Mental Health Services, Fundació Sant Joan de Déu, Dr. Antoni Pujades, 42, Sant Boi de Llobregat (Barcelona) 08830, Spain.

\section{REFERENCES}

1. Briggs AH. Handling uncertainty in economic evaluation and presenting the results. In: Drummond M, McGuire A, eds. Economic evaluation in health care: Merging theory with practice. New York, NY: Oxford University Press; 2001:172214.

2. Brooks R. EuroQol: The current state of play. Health Policy. 1996;37:53-72.

3. Covell NH, Jackson CT, Evans AC, Essock SM. Antipsychotic prescribing practices in Connecticut's public mental health sys- tem: Rates of changing medications and prescribing styles. Schizophr Bull. 2002;28:17-29.

4. Deeks JJ, Dinnes J, D'Amico R, et al. Evaluating nonrandomised intervention studies. Health Technol Assess. 2003; 7:1-173.

5. Devlin N, Parkin D. Does NICE have a cost-effectiveness threshold and what other factors influence its decision? A binary choice analysis. Health Econ. 2004:13;437452.

6. Haro JM, Edgell ET, Jones PB, et al. The European Schizophrenia Outpatient Health Outcomes (SOHO) study: Rationale, methods and recruitment. Acta Psychiatr Scand. 2003;107:222232.

7. Haro JM, Edgell ET, Novick D, et al. Effectiveness of antipsychotic treatment for schizophrenia: 6-month results of the PanEuropean Schizophrenia Outpatient Health Outcomes (SOHO) study. Acta Psychiatr Scand. 2005;111:220-231.

8. Lehman AF, Lieberman JA, Dixon LB, et al. Practice guideline for the treatment of patients with schizophrenia, second edition. Am J Psychiatry. 2004;161(Suppl):1-56.

9. McKee M, Britton A, Black N, et al. Methods in health services research: Interpreting the evidence: Choosing between randomised and non-randomised studies. BMJ. 1999;319:312315.

10. Manning WG, Mullahy J. Estimating log models: To transform or not to transform? J Health Econ. 2001;20:461494.

11. Monthly Index of Medical Specialities (MIMMS). London: Haymarket Medical Publications; February 2005.

12. Netten A, Curtis L. Unit costs of health and social care 2002. Canterbury, Kent: University of Kent Personal Social Services Research Unit; 2002.

13. Netten A, Curtis L. Unit costs of health and social care 2003. Canterbury, Kent: University of Kent Personal Social Services Research Unit; 2003.

14. Netten A, Curtis L. Unit costs of health and social care 2004. Canterbury, Kent: University of Kent Personal Social Services Research Unit; 2004.

15. Park R. Estimation with heteroscedastic error terms. Econometrica. 1966;34:888.

16. Raikou M, Briggs A, Gray A, McGuire A. Centre-Specific or average unit costs in multi-centre studies? Some theory and simulation. Health Econ. 2000;9:191-198.

17. Rosenbaum PR. Observational studies. London: Springer; 2002 .

18. Santos Silva JMC, Tenreyro S. The log of gravity. CEPR Discussion Paper Series, No. 5311; 2005.

19. Willke RJ, Glick HA, Polsky D, Schulman K. Estimating country-specific cost-effectiveness from multinational clinical trials. Health Econ. 1998;7:481-493. 